\title{
OPPORTUNISTIC SENSING NETWORKS A Study in Amsterdam
}

\author{
Adapted from "Hydrometeorological \\ Monitoring Using Opportunistic \\ Sensing Networks," by Lotte W. \\ de Vos (KNMI \& Wageningen \\ University \& Research), Arjan M. \\ Droste, Marjanne J. Zander, Aart \\ Overeem, Hidde Leijnse, Bert G. \\ Heusinkveld, Gert-Jan Steeneveld, \\ and Remko Uijlenhoet. Published \\ online in BAMS, February 2020. \\ For the full, citable article, see \\ DOI:10.1175/BAMS-D-19-0091.1.
}

I raditionally, hydrologists, meteorologists, and other scientists and practitioners have relied on dedicated measurement equipment. Such instruments, typically owned and operated by government agencies, are installed and maintained according to (inter)national standards. They offer accurate and reliable information about the state of the environment, but often lack the required spatial and/or temporal density for high-resolution monitoring or forecasting, particularly in urban areas.

Yet, with rapid development in wireless communications, sensors are now omnipresent. These are opportunistic sensors-not installed with intention to generate large-scale weather observations. While generally not as accurate or reliable as the dedicated equipment we are used to, opportunistic sensors are typically available in large numbers, and their measurements are often readily accessible online. When combined with smart retrieval algorithms and statistical treatment, these sensors could greatly benefit environmental monitoring, science, and management. Here we make a 17-day analysis for the Amsterdam metropolitan area (the Netherlands) by employing opportunistic sensors to complement conventional dedicated equipment. Our aim is to showcase the availability and meteorological capability of several opportunistic sensing techniques. 


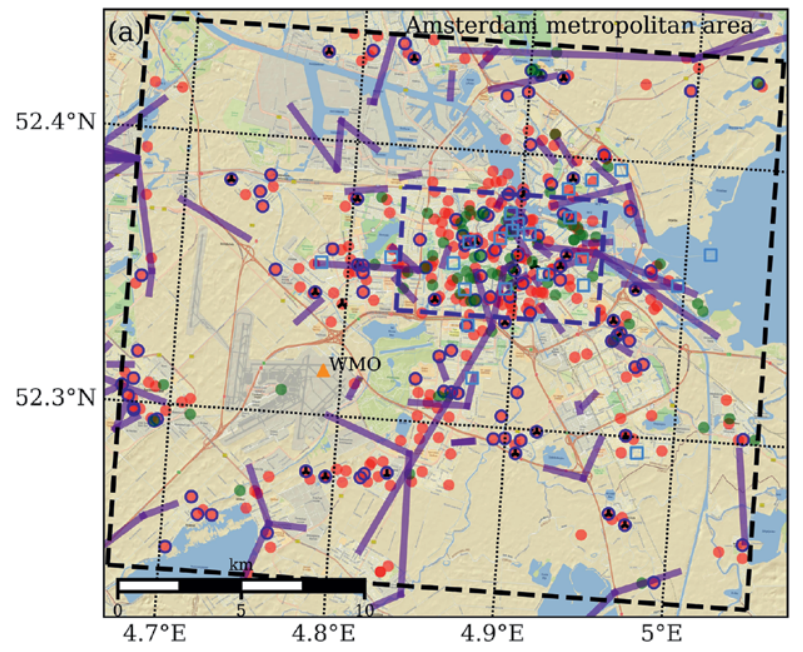

\section{Opportunistic data sources}

The opportunistic data come from three sources: smartphone sensors, commercial microwave links for cellular communications, and crowdsourced personal weather stations. We use three traditional data sources as reference for the opportunistic observations: a gauge-adjusted radar product, the WMO station at Amsterdam airport, and the Amsterdam Atmospheric Monitoring Supersite (AAMS) urban network.

Smartphones include sensors for light levels to adjust screen brightness, pressure sensors to complement the GPS for an accurate (vertical) location estimation, and thermometers for the battery to avoid damage from overheating. We utilized these sensors on smartphones for meteorological purposes. Pressure and light can be used directly, with the caveat that pressure depends on the location of the smartphone with respect to the ground. Battery temperature readings cannot be directly used as air temperature data because smartphones are often carried close to the owner's body-a heat source. We derive air temperatures from these data by using a simple two-parameter thermodynamic model trained on independent temperature data. A total of 3.14 million smartphone observations from the OpenSignal (Android) application are available with a 15-s resolution for the entire study period for the Amsterdam region. In order to reduce noise in smartphone-based data, it is necessary to aggregate in both time and space. For a city, this is typically over local climate zones and 1-h or 24-h periods.

The network supporting cell phone communication typically propagates radio signals between antennas several kilometers apart and

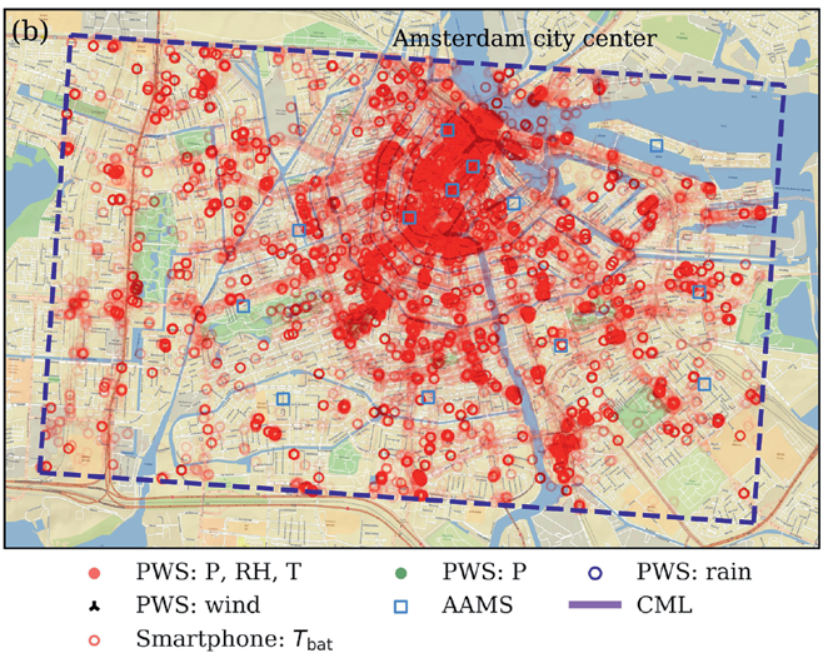

\begin{abstract}
* (a) Sensor networks in the Amsterdam metropolitan area (dashed black rectangle): personal weather stations (PWSs), commercial microwave links (CMLs), and the WMO station at the airport; (b) networks in the city center area (dashed blue rectangle), including smartphone battery temperature readings and Amsterdam Atmospheric Monitoring Supersite (AAMS) stations. Opportunistic sensors such as PWSs and smartphones are much more densely distributed than dedicated sensors (e.g., AAMS urban network).
\end{abstract}

commonly uses signal frequencies sensitive to hydrometeors. This causes attenuation of the commercial microwave link (CML) signals when liquid precipitation occurs between the antennas. This is promising because CML networks are widespread, including in areas of the world with very limited traditional rainfall sensors. Recent research has focused on improving the techniques to obtain accurate rainfall estimates and produce real-time rainfall maps. Here we use the CML network operated by T-Mobile in Amsterdam (74 links) for rainfall estimation, using the recent RAINLINK processing package.

Many low-cost personal weather stations (PWSs) can upload measurements directly to online platforms such as Netatmo Weathermap (https://weathermap.netatmo.com) to be visualized and shared in real time. Ideally, weather variables can be crowdsourced from such platforms at far higher spatial and temporal resolutions than from traditional sensor networks. However, PWSs are often installed by citizens without expertise in sensor placement and/or are subject to interference from surroundings. Hence, we expect that many 


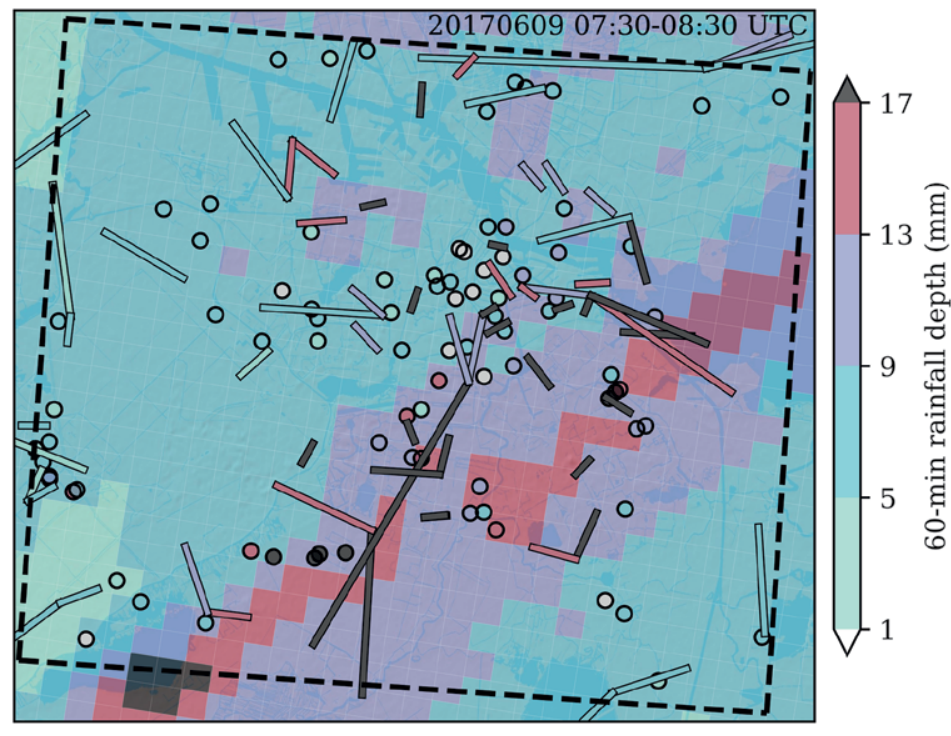

PWSs generate compromised measurements. We evaluated measurements from all PWSs from Netatmo in the Amsterdam study area. All measure temperature, pressure, and humidity, and some measure rain and/or wind, which require an additional sensor module.

We selected a case study period between 0000 UTC 6 June and 0000 UTC 23 June 2017 (local time is UTC $+2 \mathrm{~h}$ ). This period contains both sufficient opportunistic sensing data and interesting meteorological events to illustrate the potential of these techniques. The selected $22 \mathrm{~km} \times 26 \mathrm{~km}$ region was divided into an urban center dataset and a suburban dataset. We will focus on two cases: the passing of an upper-air disturbance and the subsequent passage of an active cold front and resulting rainfall on 9 June, and a hot period from 16 to 21 June that ended with the passing of a cold front on 22 June.

\section{Frontal passage}

We expect frontal passages to be distinguishable in the crowdsourced pressure and wind data. Crowdsourced and reference wind speed steadily increases as the front passes (from 2 to more than $4 \mathrm{~km} \mathrm{~h}^{-1}$ ), before reaching its maximum

(a) Median temperature measurements for $* D$ each data source. (b) The urban heat island as shown by temperature differences between median PWS city center and suburban temperatures (red dashed), the AAMS and WMO airport station (blue dashed), and the PWS center and WMO (green dashed). Shaded areas indicate nighttime.
4 * 60-min rainfall for the Amsterdam metropolitan area based on gauge-adjusted radar data (pixels; $100 \%$ availability), CML data (paths; only CMLs with $100 \%$ availability are shown), and PWS data (circles; only PWSs with at least $\mathbf{8 3 . 3 \%}$ availability are shown). The relative rainfall bias of CMLs at least $\mathbf{2}$ km long was much less than shorter links, likely due to the relatively larger contribution of error from wet antennas for short links.

$\left(5 \mathrm{~km} \mathrm{~h}^{-1}\right)$ directly after the passage. The convection associated with the upper-air disturbance at around 0300-0400 UTC generates a strong peak in the wind speed. Despite the unknown measurement setup of the PWS anemometers, the average signal of all PWSs corresponds well to that of the quality-controlled reference AAMS network (mean bias of $0.4 \mathrm{~km} \mathrm{~h}^{-1}$ ), which shows the same behavior for the upper-air disturbance and the front passing.

The ambient air pressure, measured by PWSs and smartphones, starts increasing at the moment the front passes (0800 UTC). Typically, air pressure decreases before a cold front, rapidly increases during the passage, and increases at a slower rate afterward. The expected drop prior to the frontal passage is not very pronounced in the measurements; there is a slight decrease in pressure between 0000 and 0200 UTC $(1.7 \mathrm{hPa}$ decrease for PWS; $3.5 \mathrm{hPa}$ for smartphone). The latter is more likely associated with the upper-air disturbance. After the frontal passage at 0800 UTC, the pressure rises, from $1,006 \mathrm{hPa}$ (PWS) to $1,008 \mathrm{hPa}$ (smartphone) up to a maximum of 1,013-1,016 $\mathrm{hPa}$ at midnight. The pressure tendency remains roughly $1 \mathrm{hPa} \mathrm{h} \mathrm{h}^{-1}$ after the front has passed.

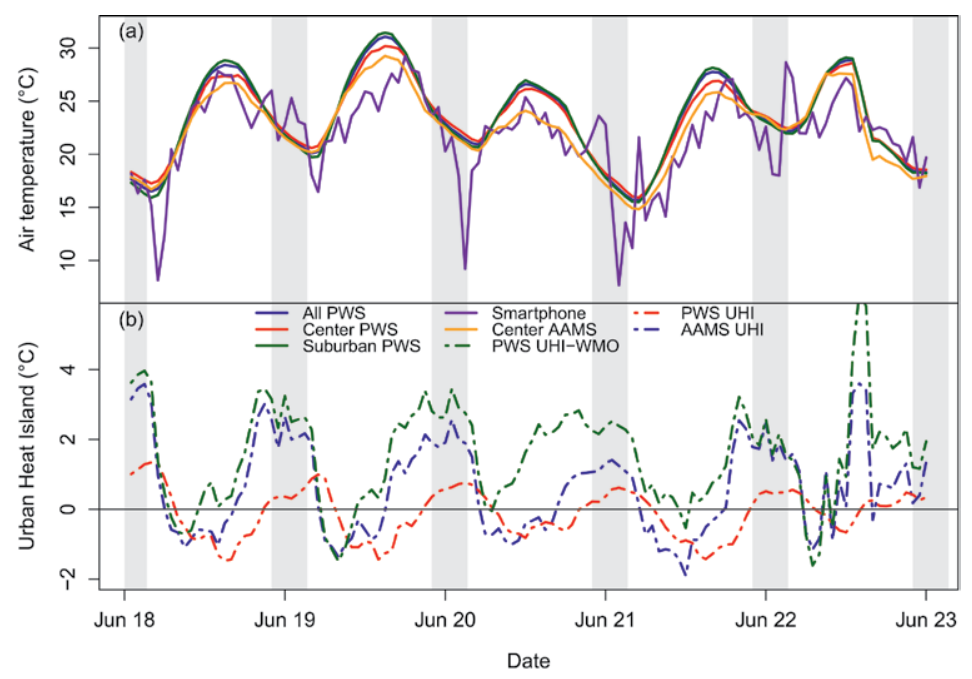


The upper-air disturbance and subsequent frontal passage of 9 June result in $27 \mathrm{~mm}$ of rainfall as measured by the gauge-adjusted radar reference. The rainfall peaks after sunrise, coinciding with the frontal passage. For the opportunistic measurements considered here, total precipitation amounts differ between the two methods, with a limited underestimation for PWSs and an overestimation for the CMLs (mainly due to short links). PWSs tend to underestimate the rain as measured by the reference, with some large reported rainfall values that are not otherwise captured. Nevertheless, the majority of PWSs seem to agree overall with the reference. The spatial and temporal distribution of rainfall measured by PWS and CML corresponds to that of the gauge-adjusted radar reference. We find that areas with the highest rainfall amounts in the reference also yield high accumulations in the CML and PWS data. Overestimation (up to $8 \mathrm{~mm}$ ) by short microwave links occurs northwest of the band. PWS observations correspond well to the spatial pattern of rain, although a number of them underestimate $(<1 \mathrm{~mm})$ it. These stations are mainly clustered in the city center. The large number of obstructions inside the city center could reduce the rainfall received by these stations.

\section{Urban heat island depiction}

The hot, generally clear, sunny weather of the last days of the study period led to higher urban temperatures and a more distinct urban heat island (UHI, here defined as the air temperature difference between city and countryside). The AAMS network serves as urban reference, and the Amsterdam airport measurements as rural reference for the UHI. The air temperature derived from smartphone battery temperatures clearly differs from the PWS and AAMS measurements, with more erratic behavior and strong minimum values at night and early morning, but better correspondence during daytime. The diurnal cycle is clearly visible: the low values at night are most likely due to a low number of measurements available, increasing the sensitivity to outliers.

The diurnal pattern of air temperature between city center and suburban PWSs is similar, although the city center stations tend to be warmer at night and colder during the day. The suburban stations contain a larger spread and bias than the center stations, though both show good agreement with the reference. The AAMS air temperature is

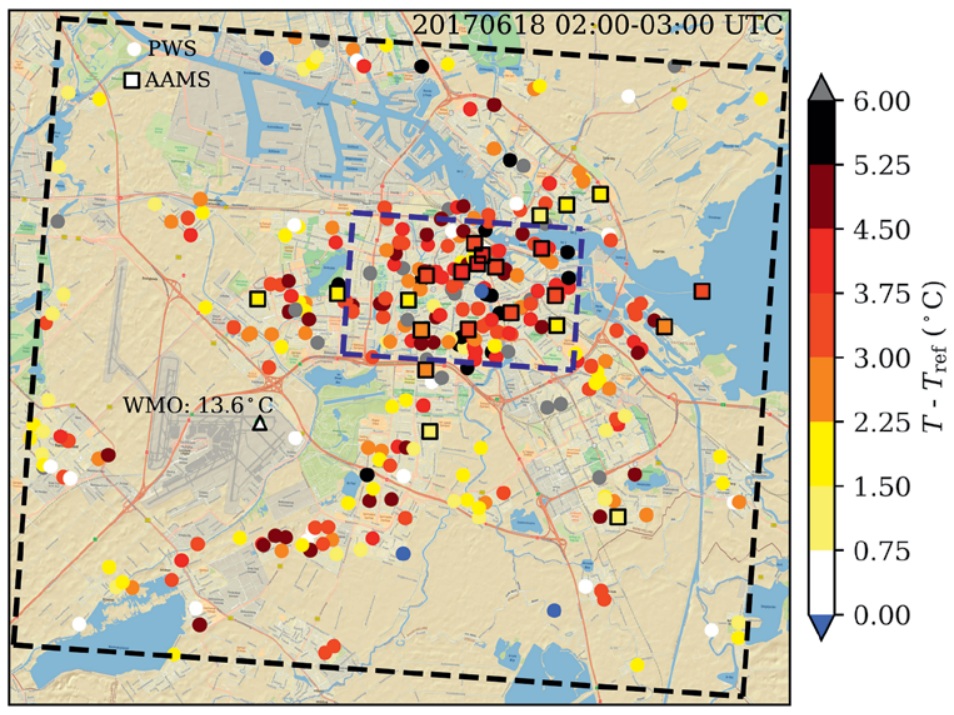

$\Delta$ between hourly averaged air temperature for AAMS network (squares) and PWSs (circles) with respect to 1.5-m air temperature at WMO station Amsterdam airport (triangle) observed at 0300 UTC. The Amsterdam metropolitan area (black rectangle) and city center (blue rectangle) are shown. Only 4 of the 309 PWSs are colder than the airport, at most by $0.6^{\circ} \mathrm{C}$, while 24 are warmer by at least $6.0^{\circ} \mathrm{C}$, at most by $12.4^{\circ} \mathrm{C}$. typically about $2^{\circ}-3^{\circ} \mathrm{C}$ lower during the day; this could partially be caused by the unknown setup of the Netatmo stations, which are likely exposed to direct sunlight or close to walls, making them sensitive to radiation errors.

\section{Outlook}

Our study shows that the opportunistic sensing techniques all yield meaningful results. However, proper quality-control procedures are needed in order for these data to become useful for many applications. PWSs are shown to perform better than smartphone or CML measurements. The PWS sensors are designed to measure hydrometeorological variables and are less reliant on quality control than the indirect CML or smartphone observations. A thorough procedure that removes error sources will therefore be most effective to improve CML and smartphone data. This may change in the future if measurement density of smartphones increases and their sensor capability improves. The opportunistic observations considered here contain large errors, as found by a larger spread in the data than would be explained by spatial or temporal variability. However, opportunistic sensors provide information on time scales and for areas that cannot be achieved with traditional sensing techniques.

We urge the scientific community to keep investigating new sources of data, and to study their uncertainties. In combination with reference networks, these new sources will provide much needed hydrometeorological information for citizens and scientists in any part of the world. 
BAMS: What would you like readers to learn from this article?

\section{Arjan Droste (Wageningen University \& Research): Opportunis-} tic sensing data is much more than just a novelty idea.

Lotte de Vos: Most of these opportunistic sensor technologies yield measurements in high spatial and temporal resolution, and/or at locations where traditional sensors are sparse. I hope this overview, with extensive reference observations to compare with, gives readers an awareness of possible alternative ways to obtain weather information, as well as an idea of the limitations.

BAMS: How did you become interested in opportunistic sensing?

LdV: For me, "opportunism" refers to getting more value out of existing infrastructure like commercial microwave links, personal weather stations from weather enthusiasts, or smartphone users. I really enjoy the idea of obtaining valuable weather information out of a dataset that was not specifically intended for that purpose. Seeing how many personal weather stations are linked to online platforms while the data were still largely unexplored, and therefore unused, motivated me to determine the actual potential of opportunistic sensors.

$\boldsymbol{A D}$ : During my university studies, I did an internship at the Dutch national weather institute. They had obtained a large dataset of smartphone data, including the battery temperature, and were interested in its potential applications to study the urban environment. We successfully managed to accurately obtain hourly urban air temperatures from such an odd data source. The power of such enormous quantities of unconventional measurements has fascinated me ever since!

\section{Gert-Jan Steeneveld (Wageningen University \& Research): Urban}

heat became a key research topic in Europe after the heat waves in 2003 and 2006. When installing professional weather stations in Dutch cities, it appeared to be extremely challenging to find representative, vandalism-proof sites where electricity was available. This made me seek personal weather stations that were already available, which offered me a rich database to make a first quantification of the urban heat island in the Netherlands.

BAMS: What surprised you the most about the work you document in this article?

GJV: I remain surprised each time / see how many variables are available via crowdsourcing. We had been working on temperature, humidity, and pressure for a while, and in this paper we find that even information about cloudiness can be retrieved using the light sensor of the smartphone.

$\boldsymbol{A D}$ : It surprised me just how well all these various opportunistic sensing techniques work together. While each individual technique has some drawback (such as the measurement quality of the smartphones, or the hard-to-correct setup errors of the PWSs), we have shown that by combining all these novel techniques we can get amazing results. The ones that are limited in area but better performance wise (like the PWS) are complemented nicely by the coarser techniques with a wide coverage such as the CML or smartphones.

LdV: The density of weather observations opportunistic sensors can yield is very promising, even if a large fraction needs to be excluded due to low quality.

BAMS: But what's remaining: do these observations have strengthor in this case, quality-in numbers?

AD: While the raw data often look like pure noise, after even some basic filtering procedures, the resulting data are already very good!

GJV: We find that crowdsourcing can only be fruitful if a strict and rigorous filtering is performed. In that sense, one should not be afraid to exclude a substantial amount of records that do not show physical behavior. If you dare to do so, we can benefit from the technique. On the other hand, the acceptance in the community of crowdsourced observations as a meaningful addition to the available observations is sometimes challenging.

BAMS: How has earning that acceptance shaped this study?

LdV: Careful exploration is needed to see how these high-quantityl low-quality observations can be used to add on our current sensor networks. Even though there exist many creative processing and filter approaches to improve accuracy of the data, we decided to mention literature describing these approaches and to show the performance of unprocessed data. Finding a way to compare them in a fair way that gives the reader good insight into the overall general potential, while keeping the article concise and to the point, was quite a challenge. 


\section{Find out from the authoritative source}

[ What's a dust devil? ]

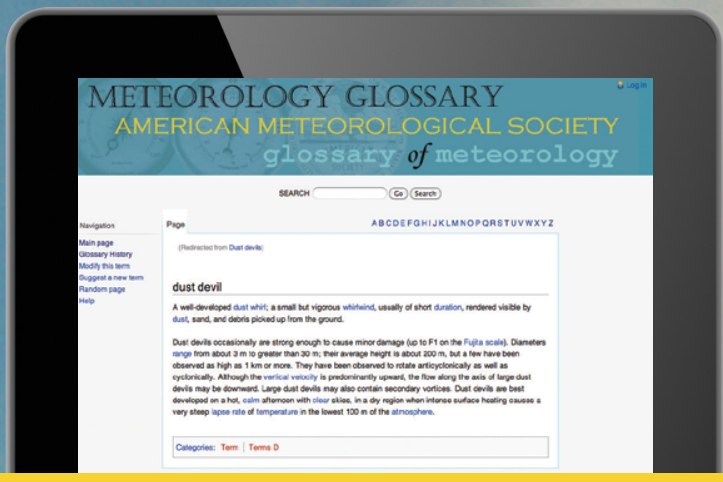

\section{Online Glossary of Meteorology}

With over 12,000 meteorological terms, you'll be able to look up definitions online any time, any place, anywhere.

http://glossary.ametsoc.org/wiki

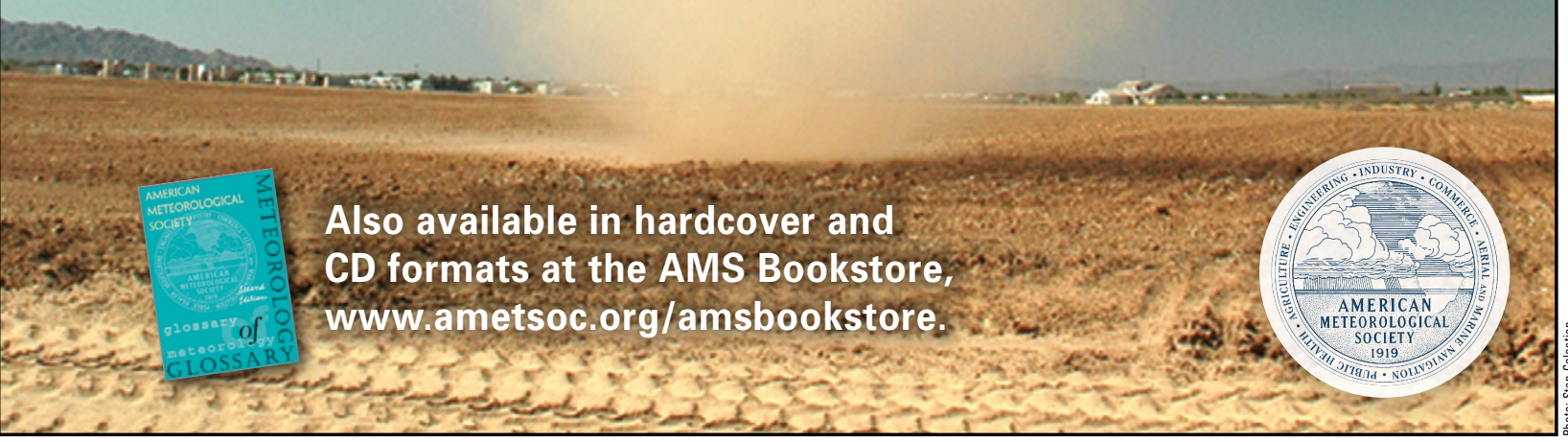

